Volume 8. No. 3, March 2020

International Journal of Emerging Trends in Engineering Research

Available Online at http://www.warse.org/IJETER/static/pdf/file/ijeter36832020.pdf

https://doi.org/10.30534/ijeter/2020/36832020

\title{
The Task of Identifying the Various Objects' Boundary Points of the Medical Image as the Optimal Path Search on the Graph
}

\author{
Pavlo Orobinskyi ${ }^{1}$, Vyacheslav Lyashenko ${ }^{2}$ \\ ${ }^{1}$ Department of Informatics, Kharkiv National University of RadioElectronics, Kharkiv, Ukraine, \\ orbpster@gmail.com \\ ${ }^{2}$ Department of Informatics, Kharkiv National University of RadioElectronics, Kharkiv, Ukraine, \\ lyashenko.vyacheslav@gmail.com
}

\begin{abstract}
The analysis and processing of medical images is an important area of research. At the same time, medical images should be regarded as the object of corresponding analysis and an increased class of complexity. One of the tasks that must be solved is the segmentation of medical images. The difficulty of solving this task lies in possible overlaying of segmentation objects or finding them at a relatively close level when they are perceived as a single object. The identification of different objects points of medical image in the form of an optimal path search on the graph is considered in this work. The formalization and substantiation of this approach have been carried out.
\end{abstract}

Key words: Segmentation, Identification, Graph, Optimal Path, Boundary Points, Medical Image.

\section{INTRODUCTION}

Image processing and analysis is widely used in various intelligent systems. For this purpose, different methods and approaches are used in the form of separate procedures [1]-[3]. This is due to the fact that such procedures allow the remote processing of information and making necessary decisions. Image is a kind of representation of the real world picture, which is formalized as a certain set of numbers, where each of these numbers transmits information. It is also possible to obtain additional information when processing the images. The additional information can be obtained by analyzing the relationship between different sets of numbers for each image.

One of the directions for using different procedures of image processing and analysis is medical information that is presented in the form of images. The analysis of such images makes it possible to diagnose the functioning of cells and tissues of various human organs, to study possible disproportions in the work of the body, and to identify diseases at an early stage. This ultimately determines the interest in the subject of this study.

\section{BRIEF ANALYSIS OF RELATED WORKS AND SELECTION OF THE MAIN RESEARCH QUESTION}

Traditionally, the application of some or other procedures for the analysis of medical images is determined by the task of a research.

For example, [4] discusses the analysis of medical images, which are cytological preparations. The authors of [4] use a wavelet ideology to analyze separate components of images of cytological preparations. At the same time, it is important to obtain additional information.

In the study presented by D. Shen, G. Wu, and H. I. Suk, it is provided a comprehensive overview of computer-assisted image analysis in the field of medical imaging [5]. Particular attention is paid to the neural network method, which has found wide application in recent image processing [6]. However, [7] emphasize the need to preserve the original information through the image analysis methods.

Y. C. Zhang and A. C. Kagen pay particular attention to the interface when creating medical image processing and analysis systems [8]. This is due to the fact that in such a process it is necessary not only to obtain the important information for subsequent decisions, but also to preserve and not to lose the original data in the application of various procedures for image analysis and processing.

It should also be considered that medical images consist of a number of individual components where one can observe the difference: 
in the size of following parts (e.g. when examining blood it can be red blood cells, white blood cells, plasma, platelets, etc.) [4]),

in the loss of information (e.g. when applying image enhancement, filtering, noise reduction procedures [1], [2]).

Thus after the loss of certain information it is necessary to concretize the results of analysis and carry out the subsequent analysis of such image.

Solving such problems leads to the fact that it is necessary to carry out the segmentation of complex objects. At the same time, such segmentation should be performed in real time in order to achieve an optimal procedure for the analysis of medical images.

Thus, the key point of this study is to consider the procedure of complex objects segmentation for medical images. In this case, the main issue of such consideration should be the identification of boundary points for each object on the medical image.

\section{EXAMPLE OF COMPLEX OBJECTS IN THE ANALYSIS OF MEDICAL IMAGES AND SPECIFICATION OF INDIVIDUAL PROBLEM POINTS}

Fig. 1 shows an example of a complex connection of individual components for a medical image that needs to be segmented.

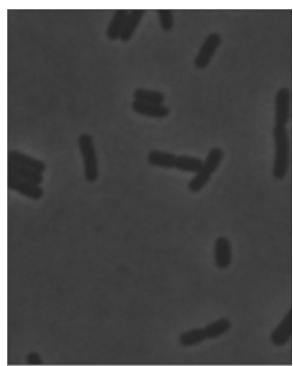

Figure 1: Example of a complex connection of individual components for a medical image that needs to be segmented

As a result of applying traditional processing methods, we get some conglomerates of objects in the image instead of individual objects. The division of such conglomerates into separate objects is the task of this study.

By the object conglomerate we mean the objects that consist of several image objects. In other words, these are the objects with brightness $\rho_{\min } \leq \rho \leq \rho_{\max }$, whereas their area significantly exceeds the maximum area of a single object in the image $-S>S_{\max }$.
The solution to this problem should be divided into two subtasks - finding the junction points of the two objects and determining the boundary of objects.

The junction point (JP) is the local extremum point (LTE), so that the function at the segment $(x-m, x)$ does not increase or decrease. Therefore, it does not stretch $(x, x+m)$, does not decrease or increase in dy, or similarly in $\mathrm{dx}$ for $(y-m, y)$ и $(y, y+m)$, where $m$ is some empirical constant (about $5 \%$ of the average length of the object circle -7 pixels). The second condition for the TS is that the object is on the side of the turned angle formed by the lines passing through the left boundary of the segment and the TLE as well as through the right boundary and the TLE (Fig. 2). Such definitions are consistent with the classical approaches in image processing [9].

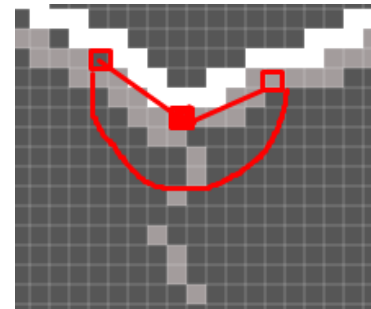

a)

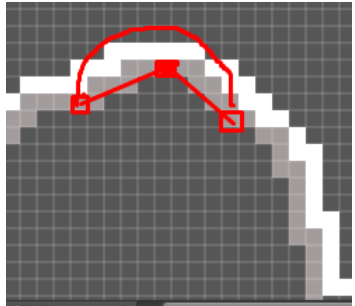

b)
Figure 2: Junction point: a) is a junction point, b) is not a junction point

The boundary refers as a sequence of 8-connected pixels connecting the starting point, i.e. the TS, with the nearest pixel belonging to the background in the given $\mathrm{R}$ direction. This is consistent with the basic approaches to image processing using classical segmentation methods [10]. The optimal point belonging to the boundary will be the point for which mathematical expectation (MO) of brightness values $5 \times 5$ of the vicinity tends to MO brightness of the boundary of the reference object (Fig. 3).

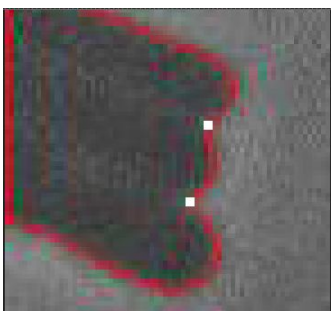

Figure 3: The junction points on the conglomerate of three objects

Such a task refers to the tasks of finding the optimal path on the $\mathrm{O}$-screen (the $\mathrm{O}$-screen is a tree because the movement direction on it is certain and two adjacent vertices should not be on the same level). Nevertheless, the problem is that the objects' boundary length of the investigated area fluctuates within 18-60 points and each processed scheme consists of 
hundreds of glued objects. Thus, the known search algorithms for the optimal path on the graph (Dijkstra, Ford-Belman, Floyd-Worschell or even the full search method [11]) are hardly applicable in practice, because the convergence of the first three methods of the order $n \wedge 2$, where $n$ is the number of vertices $n=m^{2}$, and the complete enumeration $3^{m-1}$, where $m$ is the number of levels in the tree. Thus, it will take about $7.3 * 10^{8}$ calculations just to determine the boundaries of 100 objects with an average boundary length of 40 pixels, which, even taking into account the modern computing power is very large to be really applied in solving practical problems.

\section{DETERMINATION OF BOUNDARY POINTS IN CONGLOMERATES OF SEVERAL OBJECTS}

Let's consider an ideal situation when the boundary of objects is absolutely clear, i.e. it does not contain any hindrances, therefore has an optimal top at each level of the tree, and, besides, such boundary is unique on the considered tree. Then you can use the algorithm that determines the optimal neighboring vertex at each step. Thus, the time of convergence of this algorithm will be equal to $3(m-1)$, where $m$ is a number of tree levels. However, in practice this form of algorithm is of little use, because the boundary of objects, although unique, but there are noises, which means that there is a probability that not at every step of the vertex it will be determined optimally.

The essence of the proposed procedure is to choose such a partition of the source tree, in which the given probability of finding the optimal path (i.e., in essence, the boundary of two objects) is achieved in a minimum number of iterations. Besides, we need an evaluation function that helps to evaluate at each step the optimality of the found boundary. As an evaluation measure, we will consider a multitude consisting of the most optimal peaks at its level:

$$
f_{\text {opt }}(x)=\left(\operatorname{opt}\left(x_{1}\right), \ldots, \operatorname{opt}\left(x_{n}\right)\right)
$$

where $\operatorname{opt}\left(x_{i}\right)$ returns the optimal value on $i$-th level.

Then, the non-optimal path can be chosen if at least one of the subgraphs on the $k_{i}$ level is disturbed, where $k_{i}$ is the last level of the $j$ subgraph, $j \in(1, m-1)$ subgraphs, because we are not interested in disturbances on the last level of the $m$ subgraph (Fig. 4).

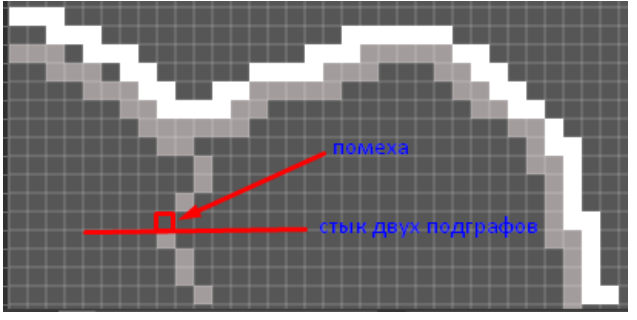

Figure 4: Example of interference at the junction of two subgraphs

Thus, the probability of interference on m-subgraphs is:

$$
\begin{aligned}
& P(a)=P_{1}(a)+P_{2}(a) \overline{P_{l}(a)}+P_{l}(a) P_{2}(a)+\ldots \\
& \ldots+P_{m-1}(a) \overline{P_{1}(a) P_{2}(a) \ldots P_{m-2}(a)}+ \\
& +P_{m-1}(a) P_{l}(a) \overline{P_{2}(a) \ldots P_{m-2}(a)}+ \\
& \quad+\ldots P_{m-1}(a) P_{1}(a) \ldots P_{m-2}(a)= \\
& =\sum_{i=1}^{m-1} C_{m-1}^{i} x^{i}(1-x)^{m-i-1}
\end{aligned}
$$

Accordingly, the probability that the chosen path is optimal in one step:

$$
P(\beta)=1-P(a)
$$

In $k$-steps, then:

$$
\begin{aligned}
& P_{k}(\beta)=P(\beta)+\overline{P(\beta)} P(\beta)+\overline{P(\beta)^{2}} P(\beta)+\ldots \\
& \ldots+\overline{P(\beta)^{k-1}} P(\beta)=P(\beta) \sum_{i=1}^{k} P(a)^{i-1}
\end{aligned}
$$

Let's look at some quantitative values (Fig. 5).

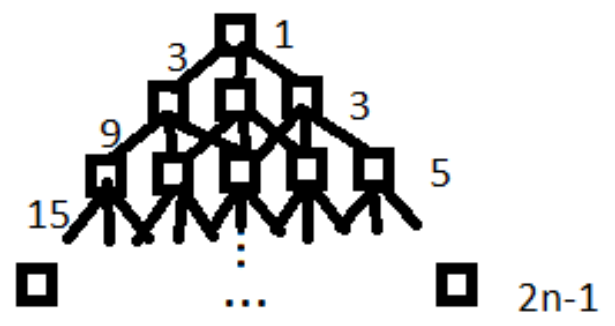

Figure 5: Tree of possible options for crossing the object border

The number of tops with possible variants in the tree is $n^{2}$, where $n$ is the number of levels in the tree. This is easy to see, since pixels have an 8-linkage, then at each step we add 2 vertices, and thus the total number of them will be equal:

$$
\begin{aligned}
& 1+3+5+\ldots+(2 i-1)+\ldots+2 n-1= \\
& =\frac{1+2 n-1}{2} n=n^{2} .
\end{aligned}
$$


Then the number of edges will be equal: $3(n-1)^{2}$. This is also not difficult to see - since there are 3 edges coming out of each vertex, except for the lower level, where all the vertices have no edges coming out, the number of edges is equal:

$$
\begin{aligned}
& m=3 n^{2}-3(2 n-1)= \\
& =3\left(n^{2}-2 n+1\right)=3(n-1)^{2} .
\end{aligned}
$$

The number of all possible track options will be determined by the formula $3^{n-1}$, which is also easy to see. Thus, for $n=2$ there may be $3=3^{l}$ different paths, for $n=3$ there will be $9=3^{2}$, and for arbitrary $n-3^{n-1}$.

We determine the optimal number of levels for each of the m-sub-platform. It will be $\frac{n}{m}$ for all subgraphs, if the remainder of the division of $\mathrm{n}$ by $\mathrm{m}$ is 0 . And $\frac{n}{m}-$ for $m-1$, $\bmod (n, m)$ for the $\mathrm{m}$-subscript, where $\bmod (n, m)$ is the residue from the division of $n$ into $m$. We will prove this statement and consider it for the case when $\bmod (n, m)=0$,

i.e. $\frac{n}{m}=k$. Then the total number of options for all segments will be equal:

$$
3^{k-1}+3^{k}(m-1)
$$

Suppose that some pair of $k-1 \mathrm{~s}$ has such a natural number $l$ that

$$
3^{k-l}+3^{k+l} \leq 2 \cdot 3^{k}, \forall l \in(1, k) .
$$

However, this statement is incorrect, as...

$$
3^{k-l}+3^{k+l} \geq 2 \cdot 3^{k} \text {. }
$$

Let us prove that

$$
\frac{3^{k-l}+3^{k+l}}{2 \cdot 3^{k}} \geq 1
$$

To do that, we will show that

$$
\left\{\begin{array}{l}
\lim _{l \rightarrow 1} \frac{3^{k-l}+3^{k+l}}{2 \cdot 3^{k}} \geq 1, \\
\lim _{l \rightarrow \infty} \frac{3^{k-l}+3^{k+l}}{2 \cdot 3^{k}} \geq 1 .
\end{array}\right.
$$

Convert the expression you are looking for.

$$
\frac{3^{k-l}+3^{k+l}}{2 \cdot 3^{k}}=\frac{3^{k-l}\left(1+3^{2 l}\right)}{2 \cdot 3^{k-l} 3^{l}}=\frac{1+3^{2 l}}{2 \cdot 3^{l}} .
$$

Then,

$$
\lim _{l \rightarrow 1} \frac{1+3^{2 l}}{2 \cdot 3^{l}}=\frac{10}{6}>1
$$

and

$$
\begin{aligned}
& \lim _{l \rightarrow \infty} \frac{1+3^{2 l}}{2 \cdot 3^{l}}=\lim _{l \rightarrow \infty} \frac{1}{2 \cdot 3^{l}}+\lim _{l \rightarrow \infty} \frac{3^{2 l}}{2 \cdot 3^{l}}= \\
& =0+\lim _{l \rightarrow \infty} \frac{3^{2}}{2}=\frac{9}{2}>1 .
\end{aligned}
$$

Proved.

Now let us show you that

$$
\left\{\begin{array}{l}
3^{k-1+l}+3^{k-l} \geq 3^{k-1}+3^{k} \\
3^{k-1-l}+3^{k+l} \geq 3^{k-1}+3^{k}
\end{array} \forall l \in(1, k-1)\right.
$$

We mean that ...

$$
\frac{3^{k-1+l}+3^{k-l}}{3^{k-1}+3^{k}} \geq 1
$$

and

$$
\frac{3^{k-1-l}+3^{k+l}}{3^{k-1}+3^{k}} \geq 1
$$

Accordingly, we get:

$$
\frac{3^{k-1+l}+3^{k-l}}{3^{k-1}+3^{k}}=\frac{3^{k-l}\left(3^{2 l-1}+1\right)}{3^{k-1}\left(3^{l-1}+3^{l}\right)}=\frac{3^{2 l-1}+1}{3^{l-1}+3^{l}},
$$

$$
\lim _{l \rightarrow 1} \frac{3^{2 l-1}+1}{3^{l-1}+3^{l}}=\frac{4}{4}=1
$$

and

$$
\begin{aligned}
& \lim _{l \rightarrow \infty} \frac{3^{2 l-1}+1}{3^{l-1}+3^{l}}=\lim _{l \rightarrow \infty} \frac{3^{2 l-1}}{3^{l-1}+3^{l}}+\lim _{l \rightarrow \infty} \frac{1}{3^{l-1}+3^{l}}= \\
& =\lim _{l \rightarrow \infty} \frac{3^{l} 3^{l-1}}{3^{l-1}(1+3)}+0=\infty>1,
\end{aligned}
$$


Pavlo Orobinskyi et al., International Journal of Emerging Trends in Engineering Research, 8(3), March 2020, 824 - 829

$$
\begin{gathered}
\frac{3^{k-1-l}+3^{k+l}}{3^{k-1}+3^{k}}=\frac{3^{2 l+1}+1}{3^{l}+3^{l+1}} . \\
\lim _{l \rightarrow 1} \frac{3^{2 l+1}+1}{3^{l}+3^{l+1}}=\frac{28}{12}>1 .
\end{gathered}
$$

and

$$
\begin{aligned}
& \lim _{l \rightarrow \infty} \frac{3^{2 l+1}+1}{3^{l}+3^{l+1}}=\lim _{l \rightarrow \infty} \frac{3^{2 l+1}}{3^{l}+3^{l+1}}+\lim _{l \rightarrow \infty} \frac{1}{3^{l}+3^{l+1}}= \\
& =\lim _{l \rightarrow \infty} \frac{3^{l} 3^{l+1}}{3^{l}(1+3)}+0=\infty>1 .
\end{aligned}
$$

It is proved that for $\bmod (n, m)=0$. This statement will also be true for the general case, where instead of 3 there will be a certain number $n$.

For $\bmod (n, m)=0$, the following partition is optimal:

$$
3^{k}(m-\bmod (n, m)+1)+3^{k+1}(\bmod (n, m)-1) .
$$

The optimality of this partitioning follows from the proven above.

Then the general formula for determining the optimal number of iterations $\operatorname{opt}(n, m)$ for the level graph $n$ divided into $m$ -subgraphs is as follows:

$$
\begin{aligned}
& \operatorname{opt}(n, m)=\left(1-\operatorname{ceill}\left(\frac{\bmod (n, m)}{\bmod (n, m)+1}\right)\right) \cdot 3^{k-1}+ \\
& +\left(m-\bmod (n, m)+2 \operatorname{ceil}\left(\frac{\bmod (n, m)}{\bmod (n, m)+1}\right)-1\right) \cdot 3^{k}+ \\
& +\left(\bmod (n, m)-\operatorname{ceil}\left(\frac{\bmod (n, m)}{\bmod (n, m)+1}\right) \cdot 3^{k+1},\right.
\end{aligned}
$$

\begin{tabular}{|c|c|c|c|c|c|c|c|c|c|c|}
\hline & 1 & 2 & 3 & 4 & 5 & 6 & 7 & 8 & 9 & 10 \\
\hline 2 & 09 & 099 & 0999 & 1 & 1 & 1 & 1 & 1 & 1 & 1 \\
\hline 3 & 081 & 0964 & 0093 & 0999 & 1 & 1 & 1 & 1 & 1 & 1 \\
\hline 4 & 0729 & 0927 & 008 & 0995 & 0999 & 1 & 1 & 1 & 1 & 1 \\
\hline 5 & 0656 & 0282 & 0059 & 0086 & 0995 & 0998 & 0999 & 1 & 1 & 1 \\
\hline 6 & 089 & 0822 & 0031 & 0972 & 0088 & 0995 & 0098 & 0999 & 1 & 1 \\
\hline 7 & 0531 & 078 & 0897 & 0052 & 097 & 0089 & 0095 & 0998 & 0999 & 0999 \\
\hline 8 & 078 & 0728 & 0858 & 0926 & 0061 & 008 & 0089 & 0995 & 0997 & 0999 \\
\hline 9 & $Q \mathcal{B}$ & 0676 & 0815 & 0895 & 094 & 0966 & 0981 & 0989 & 0994 & 0996 \\
\hline 10 & 0387 & 0625 & 077 & 0189 & 0914 & 0947 & 0068 & 098 & 0088 & 0993 \\
\hline
\end{tabular}

where ceil returns the nearest integer, that is.

$$
\left\{\begin{array}{l}
\operatorname{ceil}\left(\frac{\bmod (n, m)}{\bmod (n, m)+1}\right)=0, \bmod (n, m)=0, \\
\operatorname{ceil}\left(\frac{\bmod (n, m)}{\bmod (n, m)+1}\right)=1, \bmod (n, m)>0 .
\end{array}\right.
$$

Table 1 (as an example) shows the probability of finding the optimal path for breaks from 2 to 10 , the number of searches from 1 to 10 , and the probability of interference 0.1 .

Table 1: The probability of finding the optimal path for breaks from 2 to 10 , the number of searches from 1 to 10 , and the probability of interference 0.1
This example shows the expediency of the considered approach to the identification of boundary points in conglomerates on medical images in the form of solving the problem by searching an optimum way on the column.

\section{CONCLUSION}

The considered theoretical approach was successfully applied in practice. So, the calculated quantity of objects on $100 \%$ of investigated images coincided with their real quantity on medical images. The software tool implementing the described algorithm is created. C++ was used as the development environment and as one of the fastest software means for today. During the testing of the proposed research, it was also noted that the optimal boundary for the conglomerate points is detected much earlier than the specified probability of the indication - on the average this index is $40 \%$ of the calculated number of iterations.

Nevertheless, the open question of further research remains the applicability of the ideas discussed above for the tasks where the boundary between two objects can exceed 60 pixels many times (this is the maximum value of the objects' boundary that the authors had to face during the research). In addition, it is connected to the tasks with high level (more than $20 \%$ ) of noise pollution.

\section{REFERENCES}

1. P. Orobinskyi, D. Petrenko, and V. Lyashenko. Novel Approach to Computer-Aided Detection of Lung Nodules of Difficult Location with Use of Multifactorial Models and Deep Neural Networks, in 15th International Conference on the Experience of Designing and Application of CAD Systems (CADSM), 2019, pp. 1-5.

https://doi.org/10.1109/CADSM.2019.8779340

2. V. Lyashenko, O. Kobylin, and Y. Baranchykov. Ideology of Image Processing in Infocommunication Systems, In 2018 International Scientific-Practical 
Conference Problems of Infocommunications. Science and Technology (PIC S\&T), pp. 47-50, 2018.

https://doi.org/10.1109/INFOCOMMST.2018.8632124

3. M. Ayaz, I. Tvoroshenko, J. H. Baker, and V. Lyashenko.

Modeling the Structure of Intellectual Means of Decision-Making Using a System-Oriented NFO Approach, International Journal of Emerging Trends in Engineering Research, Vol. 7, no. 11, pp. 460-465, 2019. https://doi.org/10.30534/ijeter/2019/107112019

4. V. Lyashenko, A. Babker, and O. Kobylin. The methodology of wavelet analysis as a tool for cytology preparations image processing, Cukurova Medical Journal, Vol. 41, no. 3, pp. 453-463, 2016. https://doi.org/10.17826/cukmedj.237468

5. D. Shen, G. Wu, and H. I. Suk. Deep learning in medical image analysis, Annual review of biomedical engineering, Vol. 19, pp. 221-248, 2017.

https://doi.org/10.1146/annurev-bioeng-071516-044442

6. N. Tajbakhsh, J. Y. Shin, S. R. Gurudu, R. T. Hurst, C. B. Kendall, M. B. Gotway, and J. Liang. Convolutional neural networks for medical image analysis: Full training or fine tuning?, IEEE transactions on medical imaging, Vol. 35, no. 5, pp. 1299-1312, 2016. https://doi.org/10.1109/TMI.2016.2535302

7. H. J. Yoon, Y. J. Jeong, H. Kang, J. E., Jeong, and D. Y. Kang. Medical image analysis using artificial intelligence, Progress in Medical Physics, Vol. 30, no. 2, pp. 49-58, 2019. https://doi.org/10.14316/pmp.2019.30.2.49

8. Y. C. Zhang, and A. C. Kagen. Machine learning interface for medical image analysis, Journal of digital imaging, Vol. 30, no. 5, pp. 615-621, 2017. https://doi.org/10.1007/s10278-016-9910-0.

9. A. Gupta, and et al.. Deep learning in image cytometry: a review, Cytometry Part A, Vol. 95, no. 4, pp. 366-380, 2019. https://doi.org/10.1002/cyto.a.23701

10. L. Rundo, and et al.. MedGA: a novel evolutionary method for image enhancement in medical imaging systems, Expert Systems with Applications, Vol. 119, pp. 387-399, 2019. https://doi.org/10.1016/j.eswa.2018.11.013

11. K. C. Ciesielski, A. X. Falcão, and P. A. Miranda. Path-value functions for which Dijkstra's algorithm returns optimal mapping, Journal of Mathematical Imaging and Vision, Vol. 60, no. 7, pp. 1025-1036, 2018. https://doi.org/10.1007/s10851-018-0793-1 